\title{
Defect index of the square of a formally self-adjoint second-order difference expression
}

\section{Guojing Ren*}

\section{"Correspondence:}

rgjmaths@gmail.com

School of Mathematics and

Quantitative Economics, Shandong

University of Finance and

Economics, Jinan, Shandong

250014, P.R. China

\begin{abstract}
This paper is concerned with the defect index of the square of a formally self-adjoint second-order difference expression with real coefficients, which, in fact, is a class of formally self-adjoint fourth-order difference expressions. Sufficient and necessary conditions for such fourth-order difference expression to be a limit-2 case, a limit-3 case, and a limit-4 case are given, with respect to the limit case of the second-order difference expression. These results parallel the well-known results of Everitt and Chaudhuri for differential expressions.
\end{abstract}

MSC: 39A10; 34B20

Keywords: formally self-adjoint difference equation; defect index; limit-point case; limit-circle case

\section{Introduction}

In this paper we discuss properties of the defect index of a class of fourth-order formally self-adjoint difference expressions, which is derived from squaring the second-order difference expression,

$$
L^{2}[x](t):=L[L[x]](t), \quad t \in \mathcal{I},
$$

where

$$
L[x](t):=-\Delta(p(t) \nabla x(t))+q(t) x(t)
$$

$\Delta$ and $\nabla$ are forward and backward difference operators, respectively, i.e. $\Delta x(t)=x(t+1)-$ $x(t)$ and $\nabla x(t)=x(t)-x(t-1)$; the discrete time interval $\mathcal{I}$ is bounded from below; without loss generality, we denote $\mathcal{I}=[0,+\infty)_{\mathbb{Z}}$; and the functions $p(t), q(t)$ are all real-valued and $p(t) \neq 0$ for $t \geq-1$.

According to the classical von Neumann theory $(c f .[1,2])$ and its generalization (cf. [3]), a symmetric operator or a non-densely defined Hermitian operator has a self-adjoint extension if and only if its positive and negative defect indices are equal and its self-adjoint extension domains have a close relationship with its defect index. So it is very important to determine the defect indices of both differential and difference expressions in the study of self-adjoint extensions.

O2014 Ren; licensee Springer. This is an Open Access article distributed under the terms of the Creative Commons Attribution License (http://creativecommons.org/licenses/by/2.0), which permits unrestricted use, distribution, and reproduction in any medium, provided the original work is properly cited. 
The problem on the defect index of the second-order formally self-adjoint linear differential expression with real coefficients

$$
\mathcal{L} y=-\left(p y^{\prime}\right)^{\prime}+q y, \quad \text { on }[a, b),
$$

was first studied by Weyl [4]. It is well known that the defect index of $\mathcal{L}$ is equal to the number of linearly independent square integrable solutions of equation $\mathcal{L} y=\lambda y$ for each $\lambda \in \mathbb{C} \backslash \mathbb{R}$, where $\mathbb{C}$ and $\mathbb{R}$ denote the sets of the complex and real numbers. Later, some authors studied the defect index of $\mathcal{L}^{2}$, which, in fact, is a class of fourth-order formally self-adjoint linear differential expressions with real coefficient, and they have obtained a few excellent results [5-8].

The study of second-order difference expressions $L$ began with Atkinson's work [9] and the properties of its defect index have been sufficiently discussed. It is well known that the defect index, say $d_{L}$ is equal to the number of linearly independent solutions which are square summable of the difference equation

$$
-\Delta(p(t) \nabla x(t))+q(t) x(t)=\lambda x(t), \quad t \in \mathcal{I}
$$

for any $\lambda \in \mathbb{C} \backslash \mathbb{R}$. The value range of defect index $d_{L}$ is one or two. The second-order difference expression $L$ is called a limit-point case at $t=+\infty$ if $d_{L}=1$, that is, (1.3) has just one solution which is square summable for any point $\lambda \in \mathbb{C} \backslash \mathbb{R}$; and it is a limit-circle case at $t=+\infty$ if $d_{L}=2$, that is, (1.3) has two linearly independent solutions which are square summable for any $\lambda$, real or complex. All difference expressions of the form (1.2) come within the limit-point, limit-circle classification which depends only on the coefficients $p$ and $q$ and not on the parameter $\lambda$. Several criteria of the limit-point and limit-circle cases have been established [10-13].

The general form of a formally self-adjoint fourth-order difference expression with real coefficients is

$$
M[x](t)=\Delta^{2}\left(p_{2}(t) \nabla^{2} x(t)\right)-\Delta\left(p_{1}(t) \nabla x(t)\right)+p_{0}(t) x(t), \quad t \in \mathcal{I},
$$

where $p_{j}$ are all real-valued for $0 \leq j \leq 2$, and $p_{2}(t) \neq 0$ for all $t \in \mathcal{I}$.

Similarly as that of the second-order difference expression $L$, the defect index, say $d_{M}$, of $M$ is equal to the number of linear independent solutions which are square summable of the difference equation

$$
\Delta^{2}\left(p_{2}(t) \nabla^{2} x(t)\right)-\Delta\left(p_{1}(t) \nabla x(t)\right)+p_{0}(t) x(t)=\lambda x(t), \quad t \in \mathcal{I}
$$

for any $\lambda \in \mathbb{C} \backslash \mathbb{R}$. For such values of $\lambda$, the difference equation (1.5) may have two, three or four linearly independent solutions in $l^{2}$ and for any particular $M$ the number of such solutions is independent of $\lambda$. So all such difference expressions as $M$ can be classified into three cases: limit-2 (limit-point), limit-3 and limit-4 (limit-circle) cases. In the limit-circle case all solutions are square summable whether $\Im \lambda$ is zero or not.

In comparison with the second-order difference expressions $L$, fewer criteria for the limit case of the difference expressions $M$ have become known. Recently, it has been shown that all values of the defect index from 2 and 4 can be realized and some criteria for the limit-point case were given in [14]. 
In this paper, we focus on the fourth-order difference expression $L^{2}$ and discuss the relationship of the limit cases between $L$ and $L^{2}$. It is worth noting that, different from the differential expression, the maximal operator generated by the difference expression $L$ or $M$ may be multi-valued, and the minimal operator may be non-dense $[15,16]$. To solve this problem, we will apply the theory of subspaces to discuss the spectral theory of such a difference expression.

The rest of the paper is organized as follows. In Section 2, some preliminary work is given, including some basic concepts and useful results as regards subspaces, and the known result of the second-order difference expression $L$ and the fourth-order difference expression $M$. In Section 3, we pay attention to the defect index of the difference expression $L^{2}$. Sufficient and necessary conditions for $L^{2}$ to be limit-2 (limit-point), limit-3 and limit-4 (limit-circle) cases are given, separately. These results parallel the Chaudhuri and Everitt's result for differential expressions [5]. In the special case $p(t) \equiv 1$, which covers a number of examples which arise in practice, we establish a criterion for both $L$ and $L^{2}$ to be a limit-point case. In the final section, i.e., Section 4, some examples are given to show that all the cases of the difference expressions $L$ and $L^{2}$ considered in Section 3 can be realized.

\section{Preliminaries}

In this section, we first recall some basic concepts and useful results about subspaces. The readers are referred to $[3,16]$.

Let $X$ be a complex Hilbert space equipped with inner product $\langle\cdot, \cdot\rangle, T$ be a linear subspace (briefly, subspace) in $X^{2}:=X \times X$, and $\lambda \in \mathbb{C}$. Denote

$$
\begin{aligned}
& D(T):=\{x \in X:(x, f) \in T \text { for some } f \in X\}, \\
& R(T):=\{f \in X:(x, f) \in T \text { for some } x \in X\}, \\
& T(x):=\{f \in X:(x, f) \in T\} .
\end{aligned}
$$

It can easily be verified that $T(0)=\{0\}$ if and only if $T$ can determine a unique linear operator from $D(T)$ into $X$ whose graph is just $T$. For convenience, we will identify a linear operator in $X$ with a subspace in $X^{2}$ via its graph.

Definition 2.1 [3] Let $T$ be a subspace in $X^{2}$.

(1) The adjoint of $T$ is defined by

$$
T^{*}:=\left\{(y, g) \in X^{2}:\langle y, f\rangle=\langle g, x\rangle \text { for all }(x, f) \in T\right\} .
$$

(2) $T$ is said to be a Hermitian subspace if $T \subset T^{*} . T$ is said to be a self-adjoint subspace if $T=T^{*}$.

(3) Let $T$ be a Hermitian subspace. $T_{1}$ is said to be a self-adjoint subspace extension (SSE) of $T$ if $T \subset T_{1}$ and $T_{1}$ is a self-adjoint subspace.

Definition 2.2 [16] Let $T$ be a subspace in $X^{2} \cdot R(T-\lambda I)^{\perp}$ is called the defect space of $T$ and $\lambda$, and $\operatorname{dim}\left(R(T-\lambda I)^{\perp}\right)$ is called the defect index of $T$ and $\lambda$.

Lemma 2.1 A Hermitian subspace $T$ in $X^{2}$ with $R(T)=X$ is a self-adjoint subspace. 
Proof It suffices to show that $T^{*} \subset T$.

For any $(y, g) \in T^{*}$, since $R(T)=X$, there exists $(h, g) \in T$. Consequently, for any $(x, f) \in$ $T$,

$$
\langle f, y\rangle=\langle x, g\rangle=\langle f, h\rangle .
$$

Again by $R(T)=X$, it follows that $h=y$, which implies that $(y, g) \in T$, and consequently, $T^{*} \subset T$. Hence, $T$ is a self-adjoint subspace.

Let $T$ and $S$ be a subspace in $X^{2}$. The product of $T$ and $S$ is defined by

$$
T S=\left\{(x, g) \in X^{2}:(x, f) \in S,(f, g) \in T\right\} .
$$

Lemma 2.2 If $T$ is a closed subspace in $X^{2}$. Then $T^{*} T$ is a self-adjoint subspace in $X^{2}$.

Proof To show the result, we introduce an operator $U$ in $X^{2}$, similarly to that given for a graph of an operator (see $[17, \$ 51])$, by putting

$$
U(x, f)=(f,-x), \quad \forall(x, f) \in X^{2} .
$$

It is clear that $U$ is a unitary operator and $U^{2}=-I$, where $I$ is the identity operator in $X^{2}$.

Since $T$ is a closed subspace in $X^{2}$, so is $U T$. Therefore, the following formula is true:

$$
X^{2}=U T \oplus T^{*} .
$$

Hence, by applying the operator $U$ and $U^{2} T=T$, we have

$$
X^{2}=T \oplus U T^{*}
$$

Now, for any $(h, 0) \in X^{2}$, it can be expressed uniquely in the form

$$
(h, 0)=(x, f)+U(y, g)
$$

or

$$
(h, 0)=(x, f)+(g,-y) \text {, }
$$

where $(x, f) \in T$ and $(y, g) \in T^{*}$. This yields

$$
h=x+g, \quad 0=f-y,
$$

and, consequently,

$$
h=\left(T^{*} T+I\right)(x),
$$

where $I$ is the identity operator in $X$. Thus, the range of $T^{*} T+I$ coincides with the whole space $X$. Therefore, by Lemma 2.1, $T^{*} T+I$ is a self-adjoint subspace in $X^{2}$ and since $T^{*} T=$ $\left(T^{*} T+I\right)-I$, one sees that $T^{*} T$ also is self-adjoint. 
Corollary 2.1 If $T$ is a self-adjoint subspace in $X^{2}$, then so is $T^{n}$, where $n \geq 2$ is any integer.

Next we introduce some notation for (1.1) and (1.2). Denote

$$
l^{2}=\left\{y=\{y(t)\}_{t=-2}^{+\infty} \subset \mathbb{C}: \sum_{t=0}^{+\infty}|y(t)|^{2}<+\infty\right\} .
$$

Clearly $l^{2}$ is a Hilbert space with the inner product

$$
\langle y, z\rangle=\sum_{t=0}^{+\infty} \bar{z}(t) y(t)
$$

where $y=z$ in $l^{2}$ if and only if $\|y-z\|=0$, i.e., $y(t)=z(t), t \in \mathcal{I}$, while $\|\cdot\|$ is the induced norm.

The Green's formula for $L$ is

$$
\sum_{t=s}^{k}\{\bar{y}(t) L[x](t)-L[\bar{y}](t) x(t)\}=\left.[x, y]_{L}\right|_{s} ^{k}, \quad s, k \in \mathcal{I},
$$

where

$$
[x, y]_{L}(t)=p(t)(\nabla \bar{y}(t) x(t)-\bar{y}(t) \nabla g(t))
$$

The corresponding maximal and pre-minimal subspaces to $L$ were defined in some existing literature (e.g., [16]) by

$$
H_{L}=\left\{(x, f) \in l^{2} \times l^{2}: f(t)=L[x](t) \text { for } t \in \mathcal{I}\right\}
$$

and

$$
H_{L, 00}=\left\{(x, f) \in H: \exists t_{0} \geq 0 \text { s.t., } x(-1)=x(0)=x(t)=0, t \geq t_{0}+1\right\},
$$

respectively, and the minimal subspace was defined by $H_{L, 0}=\bar{H}_{L, 00}$, where $\bar{H}_{L, 00}$ is the closure of $H_{L, 00}$. It has been shown in [16] that $H_{L}$ may be multi-valued, and $H_{L, 00}$ and $H_{L, 0}$ are only non-densely defined Hermitian operators in $l^{2}$.

A sufficient condition for $L$ to be a limit-point case has been given.

Lemma 2.3 [10] L is a limit-point case at $t=+\infty$ if

$$
\sum_{t=0}^{+\infty} \frac{1}{|p(t)|}=+\infty
$$

A complete characterization of self-adjoint extension of $H_{L, 0}$ has been given in terms of boundary conditions. Here we recall one result which will be used.

Lemma 2.4 [16] Assume that $L$ is a limit-point case at $t=+\infty . H_{L, 1}$ is a SSE of $H_{L, 0}$ if and only if there exist real numbers $a$ and $b$ with $a^{2}+b^{2} \neq 0$ such that

$$
H_{L, 1}=\{(x, f) \in H: a x(-1)+b \nabla x(0)=0\} .
$$


Next, we consider the fourth-order formally self-adjoint difference expressions $M$. The Green formula for $M$ is

$$
\sum_{t=s}^{k}\{\bar{y}(t) M[x](t)-M[\bar{y}](t) x(t)\}=\left.[x, y]_{M}\right|_{s} ^{k}, \quad s, k \in \mathcal{I}
$$

where

$$
\begin{aligned}
{[x, y]_{M}(t):=} & \sum_{j=1}^{2}\left(\sum_{k=j}^{2}(-1)^{k-j} \Delta^{k-j}\left[p_{k}(t) \nabla^{k} \bar{y}(t)\right]\right) \Delta^{j-1} x(t-j) \\
& -\sum_{j=1}^{2} \Delta^{j-1} \bar{y}(t-j)\left(\sum_{k=j}^{2}(-1)^{k-j} \Delta^{k-j}\left[p_{k}(t) \nabla^{k} x(t)\right]\right) .
\end{aligned}
$$

The corresponding maximal and pre-minimal subspaces to $M$ are defined as follows:

$$
\begin{aligned}
& H_{M}=\left\{(x, f) \in l^{2} \times l^{2}: f(t)=M[x](t) \text { for } t \in \mathcal{I}\right\}, \\
& H_{M, 00}=\left\{(x, f) \in H_{M}: \exists t_{0} \geq 1 \text { s.t., } x(t)=0, t \in[-2,1]_{\mathbb{Z}} \cup\left[t_{0},+\infty\right)_{\mathbb{Z}}\right\},
\end{aligned}
$$

and the minimal subspace is defined by $H_{M, 0}=\bar{H}_{M, 00}$. The following is a characterization of self-adjoint extension of $H_{M, 0}$ in the case when $M$ is a limit-point case at $t=+\infty$.

Lemma 2.5 [18] Assume that $M$ is a limit-point case at $t=+\infty . H_{M, 1}$ is a SSE of $H_{M, 0}$ if and only if there exists a matrix $G_{2 \times 4}$, satisfying $\operatorname{rank} G=2, G J G^{*}=0$, and

$$
H_{M, 1}=\left\{(x, f) \in H_{M}: G u(0)=0\right\},
$$

where

$$
u(t)=\left(\begin{array}{c}
x(t-1) \\
\nabla x(t-1) \\
-\left[\Delta\left(p_{2}(t) \nabla^{2} x(t)\right)+p_{1}(t) \nabla x(t)\right] \\
p_{2}(t) \nabla^{2} x(t)
\end{array}\right), \quad J=\left(\begin{array}{cc}
0 & -I_{2} \\
I_{2} & 0
\end{array}\right),
$$

and $I_{2}$ is the $2 \times 2$ identity matrix.

Finally, in this section, we make it clear that $L^{2}$ is a special case of the fourth-order difference expressions $M$. In fact, from (1.2), one has

$$
L[x](t)=a(t+1) x(t+1)+b(t) x(t)+a(t) x(t-1)
$$

where

$$
a(t)=-p(t), \quad b(t)=p(t+1)+p(t)+q(t) .
$$

Further, applying $L$ to (2.3), one has

$$
L^{2}[x](t)=A(t+2) x(t+2)+B(t+1) x(t+1)+C(t) x(t)+B(t) x(t-1)+A(t) x(t-2),
$$


where

$$
\begin{aligned}
& A(t)=p(t) p(t-1), \\
& B(t)=-p(t)[p(t+1)+p(t-1)+2 p(t)+q(t)+q(t-1)], \\
& C(t)=p^{2}(t)+p^{2}(t+1)+(p(t+1)+p(t)+q(t))^{2} .
\end{aligned}
$$

Further, one has by formula (2.5) in [14] that

$$
L^{2}[x](t)=\Delta^{2}\left(p_{2}(t) \nabla^{2} x(t)\right)-\Delta\left(p_{1}(t) \nabla x(t)\right)+p_{0}(t) x(t),
$$

where

$$
\begin{aligned}
& p_{2}(t)=p(t) p(t-1) \\
& p_{1}(t)=p(t)[-p(t+1)-p(t-1)+2 p(t)+q(t)+q(t-1)], \\
& p_{0}(t)=q^{2}(t)+p(t) \nabla q(t)-p(t+1) \Delta q(t) .
\end{aligned}
$$

\section{Main results}

In this section, we first establish a sufficient and necessary condition for $L^{2}$ to be limitcircle case.

Theorem 3.1 $L^{2}$ is a limit-circle case at $t=+\infty$ if and only if $L$ is a limit-circle case at $t=+\infty$.

Proof We first consider the sufficiency. Suppose that $L$ is a limit-circle case at $t=+\infty$. Choose a complex number $\mu$ such that the roots of $\lambda^{2}=\mu$, say $\lambda_{1}$ and $\lambda_{2}$, are distinct. For each $j=1,2$, let $x_{1}^{j}, x_{2}^{j}$ be a fundamental set of solution of the difference equation

$$
L x(t)=\lambda_{j} x(t), \quad t \in \mathcal{I}
$$

It can easily be verified that $x_{k}^{j}, k=1,2, j=1,2$, form a fundamental set of solutions of

$$
L^{2}[x](t)=\mu x(t), \quad t \in \mathcal{I} .
$$

Since $L$ is a limit-circle case at $t=+\infty, x_{k}^{j} \in l^{2}, k=1,2, j=1,2$. Hence, $L^{2}$ is a limit-circle case at $t=+\infty$.

Next we consider necessity. If $L^{2}$ is a limit-circle case at $t=+\infty$, we can choose $\mu$ and $\lambda_{j}$ as above and conclude that all solutions of difference equation $L[x](t)=\lambda_{1} x(t)$ are in $l^{2}$. Therefore, $L$ is a limit-circle case at $t=+\infty$. The proof is complete.

The following is a direct conclusion derived from Theorem 3.1.

Corollary 3.1 $L^{2}$ is a limit-point case or limit-3 case at $t=+\infty$ if and only if $L$ is a limitpoint case at $t=+\infty$.

Theorem 3.1 completely describes the limit classification of $L^{2}$ in the limit-circle case. So we will assume, for the rest of this paper, that $L$ is a limit-point case at $t=+\infty$. In addition, 
we will assume, in the following of this paper, that $\mu$ is complex with $\Im \mu \neq 0$, and that $\lambda_{j}$, $j=1,2$, are the two distinct complex roots of $\lambda^{2}=\mu$ with $\Im \lambda_{j} \neq 0$ for $j=1,2$. Such a choice of $\mu$ is always possible.

A sufficient and necessary condition for $L^{2}$ to be a limit-point case is obtained.

Theorem 3.2 Let $L$ be a limit-point case at $t=+\infty$. $L^{2}$ is a limit-point case at $t=+\infty$ if and only if

$$
x \in l^{2}, \quad L^{2} x \in l^{2} \quad \text { implies that } \quad L x \in l^{2} .
$$

Proof First, we consider the sufficiency. Suppose that (3.3) holds.

Consider then the two difference equations (3.1) for $j=1,2$. Since $L$ is in the limit-point case at $t=+\infty$ and each $\Im \lambda_{j} \neq 0$, there will be two linearly independent solutions, $\phi_{j}$ and $\psi_{j}$, of (3.1) which satisfy $\phi_{j} \in l^{2}$ and $\psi_{j} \notin l^{2}$. In addition, by repeated application of $L$ to (3.1), we see that $\phi_{j}$ and $\psi_{j}$ are also solutions of (3.2).

Since $\lambda_{i} \neq \lambda_{j}$ with $i \neq j$, it can easily be shown that these four solutions, $\left\{\phi_{j}, \psi_{j}\right\}_{j=1}^{2}$, of (3.2) are linearly independent on $\mathcal{I}$ and so forms a basis of solutions for (3.2).

Suppose now that the result to be proved is not true, i.e. that $L^{2}$ is not a limit-point case at $t=+\infty$. Then, from the Titchmarsh-Weyl theory of difference equations [19] or [20, 21 ], it follows that (3.2) must have exactly three linearly independent solutions in $l^{2}$. Since $\left\{\phi_{j}, \psi_{j}\right\}_{j=1}^{2}$ is a basis of solutions for (3.2) and since $\phi_{j} \in l^{2}$ for $j=1,2, \ldots, n$, it follows that there must be at least one linearly independent solution, say $\psi$, of (3.2) which is of the form

$$
\psi=c_{1} \psi_{1}+c_{2} \psi_{2}
$$

such that not all the $c_{j}$ are zero and $\psi \in l^{2}$.

Since $\psi$ is a solution of (3.2), it follows that $L^{2} \psi \in l^{2}$. So by the assumption (3.3), $L \psi \in l^{2}$. So we have from this and the fact that $\psi \in l^{2}$,

$$
c_{1} \psi_{1}+c_{2} \psi_{2} \in l^{2}, \quad c_{1} \lambda_{1} \psi_{1}+c_{2} \lambda_{2} \psi_{2} \in l^{2}
$$

Since the matrix

$$
\left(\begin{array}{cc}
1 & 1 \\
\lambda_{1} & \lambda_{2}
\end{array}\right)
$$

is non-singular, one can see from (3.4) that $c_{j} \psi_{j} \in l^{2}$ for each $j=1$, 2. Since $\psi_{j} \notin l^{2}$ for all such $j$, it then follows that $c_{1}=c_{2}=0$ and this is a contradiction with the assumption. Hence $L^{2}$ is a limit-point case at $t=+\infty$.

Next we consider the necessity. Suppose that $L^{2}$ is a limit-point case at $t=+\infty$. Since $L$ is a limit-point case at $t=+\infty$, by Lemma $2.4, H_{L, 1}$, defined by (2.1), is a self-adjoint subspace extension of $H_{L, 0}$.

Consider the square $H_{L, 1}^{2}$, of the self-adjoint subspace $H_{L, 1}$. It is known by Corollary 2.1 that $H_{L, 1}^{2}$ is also a self-adjoint subspace in $l^{2} \times l^{2}$, which can be characterized by

$$
\begin{aligned}
H_{L, 1}^{2}= & \left\{(x, f) \in l^{2} \times l^{2}: L x \in l^{2}, L^{2} x=f, a x(-1)+b \nabla x(0)=0,\right. \\
& \quad \text { and } a L[x](-1)+b \nabla L[x](0)=0\} .
\end{aligned}
$$


On the other hand, consider the subspace $H_{L^{2}, 1}$, generated by $L^{2}$ and the boundary conditions

$$
\begin{aligned}
H_{L^{2}, 1}= & \left\{(x, f) \in l^{2} \times l^{2}: L^{2} x=f, a x(-1)+b \nabla x(0)=0,\right. \\
& \text { and } a L[x](-1)+b \nabla L[x](0)=0\} .
\end{aligned}
$$

A calculation shows that $H_{L^{2}, 1}$ is a self-adjoint subspace derived from $L^{2}$. In fact, by taking $G=\left(g_{i j}\right)_{2 \times 4}$ with

$$
\begin{aligned}
& g_{11}=a, \quad g_{12}=b, \quad g_{13}=0, \quad g_{14}=\frac{b}{p(1) p(0)}, \\
& g_{21}=a q(-1)-b(q(0)-q(-1)), \\
& g_{22}=[a(p(-1)+p(0))+b(2 q(0)+q(1)+4 p(0)-2 p(1)-p(-1))], \\
& g_{23}=\frac{b}{p(0)}, \\
& g_{24}=-\frac{a}{p(1)}+b \frac{2 q(0)+q(-1)+4 p(0)-2 p(-1)}{p(0) p(1)},
\end{aligned}
$$

one can verify that $\operatorname{rank} G=2, G J G^{*}=0$, and $G u(0)=0$. So by Lemma $2.5, H_{L^{2}, 1}$ is a selfadjoint subspace derived from $L^{2}$.

Thus, $H_{L, 1}^{2}$ and $H_{L^{2}, 1}$ are both self-adjoint subspaces derived from $L^{2}$ with $H_{L, 1}^{2} \subset H_{L^{2}, 1}$. Since no self-adjoint subspace can have a strict self-adjoint restriction, one must have $H_{L, 1}^{2}=H_{L^{2}, 1}$. If now we compare $D\left(H_{L, 1}^{2}\right)$ and $D\left(H_{L^{2}, 1}\right)$, we see that $x \in D\left(H_{L^{2}, 1}\right)$ implies that $L x \in l^{2}$.

For any $x \in l^{2}$ satisfying $L^{2} x \in l^{2}$, we can redefine (if necessary) the values of $x$ on $[-2,2]_{\mathbb{Z}}$, which are finite points, so that the boundary conditions in (3.5) are satisfied. Let the new function be $\tilde{x}$. Then $\tilde{x} \in D\left(H_{L^{2}, 1}\right)$ and consequently, $L \tilde{x} \in l^{2}$. Since $L x$ and $L \tilde{x}$ are different at finite points, it follows that $L x \in l^{2}$. Hence, condition (3.3) holds.

The whole proof is complete.

By the proof of Theorem 3.2, one can find some relationship of solutions of equations (3.1) and (3.2). Next, by exploiting this relationship, we prove a result which gives a necessary and sufficient condition for $L^{2}$ to be a limit-3 case at $t=+\infty$.

Theorem 3.3 Assume that $L$ is a limit-point case at $t=+\infty$. Let $\psi_{j} \notin l^{2}$ be a solution of the equation $L x=\lambda_{j} x$ for $j=1,2$. Then $L^{2}$ is a limit-3 case at $t=+\infty$ if and only if there exists a unique constant $k \neq 0$, such that

$$
\psi_{1}+k \psi_{2} \in l^{2}
$$

Proof Here it is worth noting that since $\psi_{1} \notin l^{2}$ and $\psi_{2} \notin l^{2}$ it follows that if (3.6) holds, then $k$ is unique and not zero.

We first consider sufficiency. Assume that (3.6) holds. Define the function $\psi(t)$ by

$$
\psi(t)=\psi_{1}(t)+k \psi_{2}(t)
$$


It can easily be verified that

$$
L \psi=\lambda_{1} \psi_{1}+k \lambda_{2} \psi_{2}, \quad L^{2} \psi=\mu\left(\psi_{1}+k \psi_{2}\right)=\mu \psi
$$

It follows that $L \psi \notin l^{2}$ since $k$ is unique and not zero, while $L^{2} \psi \in l^{2}$. Now if $L^{2}$ was in the limit-point case, then it would follow from Theorem 3.2 that $L \psi \in l^{2}$, which is a contradiction. In addition, it follows from Theorem 3.1 that $L^{2}$ is not a limit-circle case. So it must be a limit- 3 case at $t=+\infty$.

Next, we consider the necessity. Suppose that $L^{2}$ is a limit-3 case at $t=+\infty$. Let $\phi_{j} \in l^{2}$ be a solution of (3.1) for $j=1,2$. Thus we get four solutions, $\phi_{1}, \phi_{2}, \psi_{1}, \psi_{2}$, of (3.2). By the discussion in the proof of Theorem 3.2, it follows that these four solutions are linearly independent on $\mathcal{I}$ and so they form a basis of solutions for (3.2). Since $L^{2}$ is a limit-3 case at $t=+\infty$, there exists exactly one solution which belongs to $l^{2}$ and is linearly independent of $\phi_{1}$ and $\phi_{2}$, say $\psi$, of (3.2), which is a linear combination of $\psi_{1}$ and $\psi_{2}$, i.e.,

$$
\psi=c_{1} \psi_{1}+c_{2} \psi_{2} \in l^{2}
$$

It is evident that $c_{1} \neq 0$ since $\psi_{2} \notin l^{2}$. Define $\tilde{\psi}=c_{1}^{-1} \psi$. Then $\tilde{\psi}$ is of form (3.6).

The whole proof is complete.

Theorem 3.4 Assume that the equation $L^{2} x=0$ on $\mathcal{I}$ has exactly three linearly independent solutions in $l^{2}$. Then $L^{2}$ is a limit-3 case at $t=+\infty$ and $L$ is a limit-point case at $t=+\infty$.

Proof Since the equation $L^{2}[x](t)=0$ has exactly three linearly independent solutions in $l^{2}$, it follows that $d_{L^{2}} \geq 3$ by [15, Corollary 5.2]. In addition, one has $d_{L^{2}} \neq 4$, otherwise, $L^{2}[x](t)=0$ would have four linearly independent solutions in $l^{2}$. Hence, $d_{L^{2}}=3$ and $L^{2}$ is a limit-3 case at $t=+\infty$, and consequently $L$ is a limit-point case at $t=+\infty$ by Corollary 3.1 .

In the special case when $p(t) \equiv 1$, we have the following result.

Theorem 3.5 Assume that $p(t) \equiv 1$ for $t \geq-1$. If $|q(t)+q(t-1)|$ is bounded on $\mathcal{I}$, then both $L$ and $L^{2}$ are a limit-point case.

Proof By (2.4) and (2.5), $L^{2}$ takes the form

$$
L^{2}(x)(t)=\Delta^{2} \nabla^{2} x(t)-\Delta\left(p_{1}(t) \nabla x(t)\right)+p_{0}(t) x(t)
$$

with

$$
p_{1}(t)=q(t)+q(t-1), \quad p_{0}(t)=q^{2}(t)-\Delta \nabla q(t) .
$$

Firstly, $L$ is a limit-point case by Lemma 2.4 , since $p(t) \equiv 1$ for $t \geq-1$. Secondly, $\mid q(t)+$ $q(t-1) \mid$ is bounded on $\mathcal{I}$ implies that condition (3.1) in [14] is satisfied (with respect to $n=2$ and $\sigma(t)=1)$. Hence, $L^{2}$ is a limit-point case by [14, Theorem 3.3]. 


\section{Examples}

We finally give some examples to show that all the cases of difference expressions $L$ and $L^{2}$ can be realized.

(1) Both $L$ and $L^{2}$ are a limit-point case at $t=+\infty$ if

$$
p(t)=1, \quad q(t)=0, \quad t \geq-1
$$

or

$$
p(t)=1, \quad q(t)=(-1)^{t} t, \quad t \geq-1 .
$$

In fact, $L$ is a limit-point case by Lemma 2.3 and $L^{2}$ is a limit-point case by Theorem 3.5.

(2) Both $L$ and $L^{2}$ are a limit-circle case at $t=+\infty$ if

$$
p(t)=q(t)=4^{t-1}, \quad t \geq 0 .
$$

$L$ is a limit-circle case by [10, Example 3.2], and consequently $L^{2}$ is a limit-circle case by Theorem 3.1.

(3) $L$ is a limit-point case and $L^{2}$ is a limit-3 case at $t=+\infty$ if

$$
p(t)=2^{t}, \quad q(t)=2^{t}, \quad t \geq-1 .
$$

We first show that $L$ is a limit-point case. The solutions of the difference equation $L x=0$ are of the form $a^{t}$ with $a$ satisfying the equation $2 a^{2}-4 a+1=0$. The two roots of this equation are

$$
a_{1}=\frac{1}{2}(2+\sqrt{2}), \quad a_{2}=\frac{1}{2}(2-\sqrt{2})
$$

and then $x_{i}(t)=a_{i}^{t}$ are two solutions of $L[x]=0$. It is evident that $x_{1} \notin l^{2}$ and $x_{2} \in l^{2}$. Thus $L$ is not a limit-circle case and so it must be a limit-point at $t=+\infty$.

Similarly, solutions of the equation $L^{2}[x]=0$ are also of the form $a^{t}$ with $a$ satisfying

$$
\left(2 a^{2}-4 a+1\right)\left(8 a^{2}-8 a+1\right)=0 .
$$

The roots of $\left(8 a^{2}-8 a+1\right)=0$ are

$$
a_{3}=\frac{1}{4}(2+\sqrt{2}), \quad a_{4}=\frac{1}{4}(2-\sqrt{2}) .
$$

If $x_{1}$ and $x_{2}$ are defined as before and $x_{j}(t)=a_{j}^{t}$ for $j=3,4$, then we can see that $x_{j} \in l^{2}$ for $j=2,3,4$, but $x_{1}$ is not in $l^{2}$. Thus equation $L^{2}[x]=0$ has just three linearly independent solutions in $l^{2}$. This implies that $L^{2}$ is not a limit-point case at $t=+\infty$.

On the other hand, a calculation shows that

$$
L\left[x_{3}\right](t)=-\frac{1}{a_{3}}\left(2 a_{3}^{2}-4 a_{3}+1\right)\left(2 a_{3}\right)^{t}, \quad t \geq 0 .
$$


Since $a_{3}^{2}-4 a_{3}+1 \neq 0$ and $\left|2 a_{3}\right|>1$, it follows that $L\left(x_{3}\right) \notin l^{2}$. This shows that $L^{2}\left[x_{3}\right] \in l^{2}$ cannot imply $L\left[x_{3}\right] \notin l^{2}$. So by Theorem $3.2, L^{2}$ is not a limit-point case. Hence, $L^{2}$ is a limit-3 case at $t=+\infty$.

\section{Competing interests}

The author declares that she has no competing interests.

\section{Author's contributions}

The author worked on the results independently.

\section{Acknowledgements}

This research was supported by the NNSF of China (Grants 11226160, 11301304, 11071143, 11101241).

Received: 24 August 2013 Accepted: 10 January 2014 Published: 30 Jan 2014

\section{References}

1. Naimark, MA: Linear Differential Operators. Part II: Linear Differential Operators in Hilbert Space. Ungar, New York (1968)

2. Weidmann, J: Linear Operators in Hilbert Spaces. Springer, New York (1980)

3. Coddington, EA: Extension theory of formally normal and symmetric subspaces. Mem. Am. Math. Soc. 134, 1-80 (1973)

4. Weyl, $\mathrm{H}$ : Über gewohnliche differentialgleichungen mit singularitaten und diezu-gehorigen entwicklungen willkurlicher funktionen. Math. Ann. 68, 220-269 (1910)

5. Chaudhuri, J, Everitt, WW: On the square of a formally self-adjoint differential expression. J. Lond. Math. Soc. (2) 1, 661-673 (1969)

6. Eastham, MSP, Zettl, A: Second-order differential expression whose squares are limit-3. Proc. R. Soc. Edinb. A 76 233-238 (1977)

7. Everitt, WW, Giertz, M: On the integrable-square classification of ordinary symmetric differential expression. J. Lond. Math. Soc. (2) 10,417-426 (1975)

8. Everitt, WW, Giertz, M: A critical class of examples concerning the integrable-square classification of ordinary differential expression. Proc. R. Soc. Edinb. A 74, 285-297 (1974/1975)

9. Atkinson, FV: Discrete and Continuous Boundary Problems. Academic Press, New York (1964)

10. Chen, J, Shi, Y: The limit circle and limit point criteria for second-order linear difference equations. Comput. Math. Appl. 42, 465-476 (2001)

11. Clark, SL: A spectral analysis for self-adjoint operators generated by a class of second order difference equations. J. Math. Anal. Appl. 197, 267-285 (1996)

12. Hinton, DB, Lewis, RT: Spectral analysis of second order difference equations. J. Math. Anal. Appl. 63, 421-438 (1978)

13. Jirari, A: Second-order Sturm-Liouville difference equations and orthogonal polynomials. Mem. Am. Math. Soc. 542, 1-136 (1995)

14. Ren, G, Shi, Y: The defect index of singular symmetric linear difference equations with real coefficients. Proc. Am. Math. Soc. 138, 2463-2475 (2010)

15. Ren, G, Shi, Y: Defect indices and definiteness conditions for a class of discrete linear Hamiltonian systems. Appl. Math. Comput. 218, 3414-3429 (2011)

16. Shi, Y, Sun, H: Self-adjoint extensions for second-order symmetric linear difference equations. Linear Algebra Appl. 434, 903-930 (2011)

17. Akhiezer, NI, Glazman, IM: Theory of Linear Operators in Hilbert Space, vol. I. Pitman, London (1981)

18. Ren, G, Shi, Y: Self-adjoint subspace extensions for discrete linear Hamiltonian systems (submitted)

19. Shi, Y: Weyl-Titchmarsh theory for a class of singular discrete linear Hamiltonian systems. Linear Algebra Appl. 416, 452-519 (2006)

20. Šimon Hilscher, R, Zemánek, P: Weyl disks and square summable solutions for discrete symplectic systems with jointly varying endpoints. Adv. Differ. Equ. 2013, 232 (2013)

21. Clark, S, Zemánek, P: On a Weyl-Titchmarsh theory for discrete symplectic systems on a half line. Appl. Math. Comput. $217,2952-2976(2010)$

10.1186/1687-1847-2014-48

Cite this article as: Ren: Defect index of the square of a formally self-adjoint second-order difference expression. Advances in Difference Equations 2014, 2014:48 